\title{
Editorials
}

\section{Another bleeding heart: perioperative heparin management revisited}

\author{
Christopher C. Harle MB ChB FRCA, John M. Murkin MD FRCPC
}

$\mathrm{H}$ EPARIN anticoagulation titrated by prolongation of activated clotting time (ACT) continues to be a cornerstone of surgery employing cardiopulmonary bypass $(\mathrm{CPB})$. Unfractionated heparin $(\mathrm{UH})$ remains the standard anticoagulant used for extracorporeal circulation in the context of $\mathrm{CPB}$, while low molecular weight heparin (LMWH) is increasingly used in the management of patients with unstable angina and in the prevention of venous thrombosis. We know that patients receiving $\mathrm{UH}$ preoperatively are at risk of demonstrating heparin resistance, or decreased heparin responsiveness, thus it does not come as a surprise that in this issue of the Journal, Bar-Yosef et al. present evidence that preoperative treatment with LMWH is also associated with decreased heparin responsiveness as measured by celite ACT. ${ }^{1}$ And there's the rub -- is there in actuality a lesser degree of anticoagulation, or does decreased heparin responsiveness in part reflect limitations in our current clinical measures of anticoagulation?

Unfractionated heparin is a linear anionic sulphated glycosaminoglycan. It is a heterogeneous compound with variability in both the length and composition of the side chain carbohydrates which explains the range of its molecular weights (MW) from 3,000 to 30,000 Da. Low molecular weight heparin consists of short chain polysaccharides, with an average molecular weight of less than 8,000 Da. The plasma half-life of LMWH is considerably longer than (two to four times) that of $\mathrm{UH}$, and is prolonged even further in renal failure. The critical high affinity binding site for antithrombin (AT) are comprised of unique sequences of pentasaccharide units which occur in only about one third of heparin chains and are randomly distributed. The primary anticoagulant effect of heparins is via activation of AT producing a heparin-antithrombin (H-AT) complex which inactivates thrombin, activated factor X (fXa) and other factors. Additionally, both UH and LMWH can be shown to have non-AT dependent anticoagulant properties via direct inhibition of 'intrinsic' tenase, a phospholipid bound complex of fVIIIa and fIXa which generates fXa. Extrinsic tenase, a complex of phospholipid bound tissue factor, $\mathrm{fX}$ and fVIIa, also generates $\mathrm{fXa}$ but is not inhibited by either UH or LMWH. ${ }^{2}$ Other, non-AT-dependent anticoagulant activity is seen at high heparin concentrations wherein activation by large MW heparin of a second plasma protein, heparin co-factor II, impairs fXa generation. Such a heparin co-factor II-mediated anticoagulant effect of heparin could be operative in severe AT deficiency. ${ }^{3}$

Large MW H-AT inhibits thrombin through a mechanism involving non-specific binding of thrombin coincident with high affinity binding of AT, whereas inhibition of fXa requires only high affinity binding of AT. ${ }^{4}$ Small heparin fragments (< 18 saccharides) lack ability to simultaneously bind AT and thrombin, thus LMWH has limited antithrombin activity (insensitivity of ACT) but relatively potent anti-Xa activity (antithrombotic properties). As such, the anticoagulant and antithrombotic properties of heparin are only weakly correlated, and it has been demonstrated that LMWH is a weaker anticoagulant than $\mathrm{UH}$ but has equal or superior antithrombotic activity. ${ }^{5}$ Hence, its increasing usage in acute coronary syndromes and related settings as in the current study.

The pharmacokinetic profile of heparin is complex, even before factoring in the effects of hypothermia, hemodilution, organ dysfunction and CPB. Males and cigarette smokers metabolize heparin faster than females and non smokers. There are many other vari-

CAN J ANESTH 2007 / 54: 1 / pp 97-102

From the Department of Anesthesiology and Perioperative Medicine, University of Western Ontario, London, Ontario, Canada.

Address correspondence to: Dr. John M. Murkin, Department of Anesthesiology, University of Western Ontario, 339 Windermere Road, London, Ontario N6A 5A5, Canada. Phone: 519-663-3384; E-mail: jmurkin@uwo.ca 
ables which affect the heparin dose response at any given time. These include the individual patient's heparin responsiveness, which in turn may be affected by AT levels, platelet numbers and function, temperature, $\mathrm{pH}$, concurrent medication including aprotinin or anticoagulant therapy, and the patient's pharmacogenetic profile and nutritional status including serum albumin. In addition, many of the heparin binding proteins are acute phase reactants such as activated protein $\mathrm{C}$, such that heparin resistance is often encountered in critically ill patients, those with malignancies and in the peripartum period. Organ dysfunction associated with critical illness, and acute coronary syndromes are further confounders.

The physiological balance between hemostasis and bleeding can be affected at many levels, by diseases, by drugs and by the inflammatory responses to injury and surgery. Sick patients have lower levels of AT. Acquired AT deficiency has been linked to critical illness, trauma and sepsis. ${ }^{6}$ In addition, preoperative heparin use and iv nitroglycerin infusion have also been reported to decrease AT. ${ }^{7}$ The patients in Bar-Yosef et al.'s study ${ }^{1}$ who were treated with UH or LMWH appear to have been sicker, and were probably in-patients. This may in part explain the decreased heparin responsiveness seen in these patients. Furthermore, the 'potency' of heparin is not consistent.

Measures of heparin potency are not standard from country to country. The potency of heparin is determined by comparing the ability of heparin to prolong coagulation with that of a known control. The United States Pharmacopoeia and British Pharmacopoeia use a different method from the European Pharmacopoeia. The World Health Organisation, in 1999, called for international standardization of heparin potency. However, this has seemingly not yet been adapted universally, and different vendors provide heparin with different units of potency, for example Hepalean ${ }^{\circledR}$ is presented with United States Pharmacopoeia references, while Heparin Leo ${ }^{\circledR}$ is presented in international units, or British Pharmacopoeia units. Further, variability exists even within batches of $\mathrm{UH}$ provided by the same vendor, presumably due to the heterogeneity of the heparin molecules.

In dosages used for CPB, (e.g., 300-400 IU. $\mathrm{kg}^{-1}$ ), UH has a plasma half-life of approximately $90 \mathrm{~min}$ with lower concentrations cleared via the reticuloendothelial system and higher concentrations via a non-saturable renal clearance. ${ }^{8}$ In the context of CPB, residual circulating heparin concentration can be estimated based on a 90-min half-life such that approximately one-half the administered dose(s) of heparin remains bioactive $90 \mathrm{~min}$ after administration. This has several clinical implications. Failure to consider heparin clearance during $\mathrm{CPB}$ can result in significantly lower heparin concentrations during $\mathrm{CPB}$ that may not be reflected in lower ACT values but still gives rise to subclinical thrombin generation and factor consumption. ${ }^{9}$ Further, since reversal of $\mathrm{UH}$ is achieved with protamine sulphate using an approximate ratio of $1 \mathrm{mg}$ protamine to $100 \mathrm{IU}$ of heparin, failure to consider heparin clearance can result in administration of significantly larger dosages of protamine than required. As protamine in its own right has anticoagulant properties, ${ }^{10}$ excess protamine can contribute to postoperative bleeding. As a highly cationic peptide, protamine binds to heparin to form a stable ion pair devoid of anticoagulant activity which is then cleared and broken down by the reticuloendothelial system. Protamine does not reverse the effects of $\mathrm{LMWH}$, and it is recognized that heparin rebound can occur even following adequate initial reversal of heparin.

Despite exponential growth in our understanding of the complex interactions modulating coagulation, we have made only limited progress in the management of anticoagulation and monitoring during CPB. The belated recognition that the kallikrein-inactivator activity of aprotinin prolongs 'extrinsic' pathway activation and thus celite-based ACT - but not tissue factor-based 'intrinsic' coagulation - lead to decreased heparin dosing and increased thrombotic complications in cardiac surgical patients. ${ }^{11}$ Similarly, the phenomenon described as 'aprotinin-induced heparin resistance' in actuality reflected increased protamine dosing based on misinterpretation of celite-based ACT values. ${ }^{12}$ So what are the limitations of ACT monitoring?

The ACT is a relatively crude and non-standardized test of anticoagulation. Primarily responsive to 'intrinsic' pathway activation, it can be confounded by the choice of activator (celite or kaolin), type of device, degree of hemodilution, extent of platelet activation, temperature of sample, concentration of aprotinin, and operator technique, even before considering actual alterations in hemostatic activity. ${ }^{13}$ Several alternatives to simple celite or kaolin based ACT monitoring have been proposed, including targeting whole blood heparin concentration, ${ }^{9}$ highdose thrombin time, plasma supplemented modified activated clotting time and an anti-Xa-activity-based coagulation assay. Despite the significant limitations of ACT monitoring including its lack of specificity and sensitivity, however, it remains the standard in many (most) centres where cardiac surgery is practiced.

There are alternatives to systemic heparinization, and currently there is much interest in the short-act- 
ing direct thrombin inhibitor, bivalirudin, an agent which can achieve effective anticoagulation for $\mathrm{CPB} .{ }^{14}$ Similarly, argatroban has been used to facilitate CPB in patients with heparin-induced thrombocytopenia antibodies undergoing cardiac surgery. ${ }^{15}$ Heparinbonded CPB circuits may have a role in reducing the need for systemic heparinization as well, however they are not used universally.

The enormous potential variability of the effects of heparin on coagulation, along with the differences in patients and their diseases, and the vagaries of the tools we use to monitor the effects of heparin render the prediction of achieving a target ACT based on simple dosing algorithms illogical and unscientific. Ideally, each patient should have a carefully thought out plan for anticoagulation for $\mathrm{CPB}$, taking into account the factors mentioned. Quantification of the heparin dose response, whether using a heparin sensitivity index as used in the report by Bar-Yosef $e t$ al., ${ }^{1}$ or by using a device such as the Hepcon ${ }^{\circledR}$, or by simply constructing a manual dose response curve for each patient might alert the physician to potential problems with decreased heparin responsiveness and should raise a red flag to the cardiac anesthesiologist, surgeon and perfusionist alike.

It is clear that too little heparin is bad, as incomplete anticoagulation results in coagulation on $\mathrm{CPB}$, with at best a consequent consumptive coagulopathy, and at worst thrombosis of the $\mathrm{CPB}$ circuit. Equally, heparin overdose is undesirable as platelet dysfunction and excessive postoperative bleeding are associated with too much heparin. In sum, we should maintain a high index of suspicion of reduced heparin responsiveness for all acutely ill patients, especially for those receiving $\mathrm{UH}$ or LMWH preoperatively as demonstrated by Bar-Yosef et al. ${ }^{1}$ in this issue of the Journal.

\section{Un autre cœur qui} saigne : une réévaluation de l'anticoagulation à l'héparine

L'anticoagulation à l'héparine titrée par la prolongation du temps de coagulation activé (TCA) demeure la pierre angulaire de la chirurgie avec circulation extracorporelle (CEC). L'héparine non fractionnée (HNF) demeure l'anticoagulant de référence utilisé pour la circulation extracorporelle, mais l'héparine de bas poids moléculaire (HBPM) est de plus en plus utilisée dans la prise en charge de patients souffrant d'angine instable et pour la prévention des thromboses veineuses. Nous sommes conscients que les patients recevant de l'HNF avant la chirurgie encourent le risque de montrer une résistance à l'héparine ou une réaction diminuée à l'héparine. Dès lors, il n'est pas surprenant que dans ce numéro du Journal, Bar-Yosef et al. fournissent des preuves que le traitement préopératoire avec l'HBPM est également lié à une réponse diminuée à l'héparine telle que mesurée par le TCA avec célite. ${ }^{1}$ Voici la difficulté : y a-t-il en fait une anticoagulation diminuée, ou bien une réponse réduite à l'héparine reflète-t-elle en partie les limites de nos mesures cliniques actuelles de l'anticoagulation ?

L'héparine non fractionnée se compose de glycosaminoglycans linéaires anioniques sulfatés. C'est un composé hétérogène dont la longueur et la composition des hydrates de carbone de la chaîne latérale sont variables, expliquant son échelle de poids moléculaire (PM) allant de 3000 à 30000 Da. L'héparine de bas poids moléculaire consiste en polysaccharides de courte chaîne, avec moyen moléculaire de moins de $8000 \mathrm{Da}$. La demi-vie plasmatique de l'HBPM est bien plus longue (deux à quatre fois) que celle de l'HNF, et se prolonge davantage en présence d'une insuffisance rénale. Le site de liaison à forte affinité pour l'antithrombine (AT) est crucial et se compose d'une séquence unique d'unités de penta-saccharides, qui n'apparaît que dans un tiers environ des chaines d'héparine et est distribuée de manière aléatoire. L'effet anticoagulant primaire des héparines a lieu par l'activation de l'AT, créant un complexe héparineantithrombine (H-AT) qui désactive la thrombine, le facteur activé X (fXa) ainsi que d'autres facteurs. En outre, l'HNF et l'HBPM présentent toutes deux des propriétés anticoagulantes indépendantes de l'AT via l'inhibition directe de la tenase "intrinsèque », celle-ci étant un complexe des facteurs fVIIIa et fIXa lié à des phospholipidiques membranaires qui génère le fXa. La tenase extrinsèque, un complexe de facteur tissulaire lié à des phospholipides, de fX et de fVIIa, génère également le fXa mais n'est inhibée ni pas l'HNF ni par l'HBPM. ${ }^{2}$ Une activité anticoagulante indépendante de l'AT est observée à de hautes concentrations d'héparine. En effet, l'activation par héparine à PM élevé d'une deuxième protéine plasmatique, l'héparine co-facteur II, entrave la production de fXa. Cet effet anticoagulant de l'héparine médié par le co-facteur II pourrait fonctionner en présence d'une déficience importante d'AT. 
Dans le cas de la HNF, le complexe H-AT inhibe la thrombine par un mécanisme impliquant une liaison non spécifique de la thrombine qui coïncide avec une forte affinité de liaison de l'AT, alors que l'inhibition du fXa nécessite seulement une forte affinité de liaison de l'AT. ${ }^{4}$ Les petits fragments d'héparine $(<18$ saccharides) ne sont pas capables de se lier simultanément avec l'AT et la thrombine; ainsi, l'HBPM présente une activité antithrombinique limitée (insensibilité du TCA) mais une activité relativement importante d'anti-Xa (propriétés antithrombotiques). A ce titre, les propriétés anticoagulantes et antithrombotiques de l'héparine ne sont que faiblement corrélées, et il a été démontré que l'HBPM est un anticoagulant plus faible que l'HNF mais qu'il possède une activité antithrombotique égale ou supérieure. ${ }^{5}$ Ceci explique son utilisation croissante dans le traitement des syndromes coronariens aigus, comme dans l'étude dont il est ici question.

Le profil pharmacocinétique de l'héparine est complexe et ce, même avant de prendre en compte les effets de l'hypothermie, de l'hémodilution, du dysfonctionnement des organes et de la CEC. Les hommes et les fumeurs métabolisent l'héparine plus rapidement que les femmes et les non fumeurs. De nombreuses autres variables influencent constamment la relation dose-réponse de l'héparine. On peut citer la sensibilité individuelle à l'héparine de chaque patient, laquelle peut être affectée par les niveaux d'AT, le décompte et la fonction des plaquettes, la température, le $\mathrm{pH}$, une médication concomitante incluant de l'aprotinine ou une thérapie anticoagulante, ainsi que le profil pharmacogénétique du patient et le statut nutritionnel, y compris l'albumine sérique. En outre, de nombreuses protéines liant l'héparine sont des réactifs de phase aiguë, comme par exemple la protéine $\mathrm{C}$ activée, ce qui a pour conséquence qu'une résistance à l'héparine est souvent observée chez des patients très malades, ceux souffrant d'un cancer ou en peripartum. L'atteinte multi-organe associée à une maladie grave ainsi que les syndromes coronariens aigus sont d'autres facteurs confondants.

L'équilibre physiologique entre l'hémostase et le saignement peut être influencé à de nombreux niveaux, par les maladies, les médicaments et les réactions inflammatoires à des lésions ou suite à une chirurgie. Les patients plus malades ont des niveaux plus bas d'AT - une déficience d'AT acquise suite à une maladie grave, un trauma ou un sepsis. ${ }^{6}$ En outre, on a également observé que l'utilisation préopératoire d'héparine et la perfusion intraveineuse de nitroglycérine réduisaient l'AT. ${ }^{7}$ Les patients de l'étude de Bar-Yosef et al. ${ }^{1}$ qui ont été traités avec de l'HNF ou de l'HBPM semblent avoir été très malades, et étaient vraisemblablement hospitalisés. Ceci pourrait expliquer en partie la réponse réduite à l'héparine observée chez ces patients. En plus, la 'puissance' de l'héparine n'est pas constante.

Les mesures de puissance de l'héparine ne sont pas internationalement standardisées. La puissance de l'héparine est déterminée en comparant la capacité de l'héparine à prolonger la coagulation et celle d'un témoin connu. Les Pharmacopées des États-Unis et de Grande-Bretagne utilisent une méthode différente de la Pharmacopée européenne. En 1999, l'Organisation Mondiale de la Santé a exigé une standardisation internationale de la puissance de l'héparine. Toutefois, cela n'a pas encore été universellement adapté, et des fournisseurs différents offrent de l'héparine présentant différentes unités de puissance. Par exemple, l'Hepalean ${ }^{\circledR}$ se présente avec les références de la Pharmacopée des États-Unis, alors que l'Heparin Leo ${ }^{\circledR}$ est vendue avec les unités internationales ou les unités de la Pharmacopée britannique. De plus, il y a même des variantes au sein d'un même lot d'HNF provenant d'un même fournisseur, soi-disant à cause de l'hétérogénéité des molécules d'héparine.

Aux doses utilisées pour la CEC (par ex. 300-400 IU. $\left.\mathrm{kg}^{-1}\right)$, l'HNF a une demi-vie plasmatique d'environ $90 \mathrm{~min}$; à des concentrations faibles, l'élimination s'effectue via le système réticulo-endothélial, tandis qu'une élimination rénale non saturable prédomine à des concentrations plus élevées. ${ }^{8}$ Dans le contexte de la CEC, la concentration résiduelle d'héparine circulante peut être estimée en se basant sur une demi-vie de 90 min : ainsi, environ la moitié de la dose d'héparine administrée demeure bioactive 90 min après l'administration. Ceci comporte de nombreuses implications cliniques. Le fait de ne pas tenir compte de l'élimination de l'héparine pendant la CEC peut avoir pour conséquence des concentrations d'héparine nettement plus faibles durant la CEC, qui ne sont pas traduites par des valeurs plus basses de TCA mais peuvent quand même générer de la thrombine en concentration sub-clinique et la consommation de facteurs. ${ }^{9}$ En outre, étant donné que la neutralisation de l'HNF est obtenue avec du sulfate de protamine à un ratio approximatif d'l $\mathrm{mg}$ de protamine pour $100 \mathrm{IU}$ d'héparine, le fait de ne pas tenir compte de l'élimination de l'héparine peut avoir comme conséquence l'administration d'une dose de protamine bien plus importante que nécessaire. La protamine présentant elle-même des propriétés anticoagulantes, ${ }^{10}$ un excès de cette dernière peut provoquer des saignements postopératoires. En tant que peptide fortement cationique, la protamine se lie 
à l'héparine pour former une paire d'ions stable qui n'a pas d'activité anticoagulante ; il est ensuite éliminé et fragmenté par le système réticulo-endothélial. La protamine est fréquemment administrée de façon empirique. La protamine ne neutralise pas les effets de l'HBPM et il est de plus reconnu qu'un rebond d'héparine peut survenir même après une neutralisation initiale adéquate de l'héparine.

Malgré la croissance exponentielle de notre compréhension des interactions complexes qui modulent la coagulation, nous avons seulement fait des progrès limités dans la gestion de l'anticoagulation et la surveillance durant la CEC. La reconnaissance tardive que l'activité inhibitrice de l'aprotinine sur la kallicréine prolonge l'activation de la voie 'extrinsèque' et de ce fait le TCA à base de célite - mais pas de la coagulation 'intrinsèque' initiés par le facteur tissulaire - a conduit à la réduction de la posologie d'héparine et accru les complications thrombotiques chez les patients de chirurgie cardiaque. ${ }^{11}$ De même, le phénomène que l'on dénomme 'résistance à l'héparine induite par l'aprotinine' reflète en fait un dosage accru de protamine basé sur une mauvaise interprétation des valeurs de TCA au célite. ${ }^{12}$ Quelles sont donc les limites du monitorage du TCA ?

Le TCA est un test du degré d'anticoagulation relativement sommaire et non standardisé. Réagissant principalement à l'activation de la voie 'intrinsèque', il peut être affecté par le choix de l'activateur (célite ou kaolin), le type d'appareil, le degré d'hémodilution, le degré d'activation plaquettaire, la température de l'échantillon, la concentration d'aprotinine et la technique de l'opérateur, et ce, même avant de considérer les modifications de l'activité hémostatique. ${ }^{13}$ Plusieurs alternatives au monitorage à base de célite ou de kaolin ont été proposées, y compris celle de cibler la concentration d'héparine dans le sang complet, ${ }^{9}$ le temps de thrombine à haute dose, un temps de coagulation activé modifié par un supplément de plasma et un test de coagulation basé sur l'activité anti-Xa. Malgré les limites que présente le monitorage par TCA, notamment son manque de spécificité et de sensibilité, ce type de monitorage demeure néanmoins la norme dans plusieurs (la plupart des) centres de chirurgie cardiaque.

Il existe des alternatives à l'héparinisation systémique; on s'intéresse actuellement beaucoup à la bivalirudine, un inhibiteur direct de la thrombine à courte action. Il s'agit d'un agent pouvant atteindre une anticoagulation efficace pour la CEC. ${ }^{14}$ De même, l'argatroban a été utilisé pour faciliter la CEC chez les patients de chirurgie cardiaque présentant des anticorps en association avec une thrombocytopé- nie provoquée par l'héparine. ${ }^{15}$ Les circuits de CEC enduits d'héparine pourraient jouer un rôle dans la réduction des besoins en héparinisation systémique; toutefois, ils ne sont pas utilisés universellement.

La variabilité potentielle gigantesque des effets de l'héparine sur la coagulation, la variété des patients et de leurs maladies ainsi que les aléas des outils que nous utilisons pour surveiller les effets de l'héparine font qu'il est illogique et non scientifique de prédire un TCA cible en se basant simplement sur des algorithmes de dosage simple. Idéalement, chaque patient devrait disposer d'un plan d'anticoagulation pour la CEC préparé avec soin, et qui tiendrait compte des facteurs mentionnés ici. La quantification de la dose réponse à l'héparine, réalisée soit à l'aide d'un indice de sensibilité à l'héparine tel qu'utilisé dans l'étude de Bar-Yosef et al. ${ }^{1}$, soit à l'aide d'un appareil comme le Hepcon ${ }^{\circledR}$, ou simplement en calculant manuellement une courbe dose-réponse pour chaque patient, pourrait prévenir le médecin de problèmes potentiels liés à une réponse diminuée à l'héparine, et devrait également alerter l'anesthésiologiste cardiaque, le chirurgien et le perfusionniste.

Il est clair qu'une dose trop faible d'héparine est mauvaise, puisqu'une anticoagulation incomplète engendre la coagulation pendant la CEC, avec pour conséquence une coagulopathie de consommation dans le meilleur des cas, et une thrombose du circuit de CEC dans le pire. De même, une surdose d'héparine est indésirable. En effet, la dysfonction plaquettaire et un saignement postopératoire excessif sont liés à une surdose d'héparine. En somme, nous devrions maintenir un haut degré de suspicion lorsque confronté à une réponse réduite à l'héparine chez tous les patients sévèrement malades et plus particulièrement chez ceux recevant de l'HNF ou de l'HBPM avant la chirurgie, comme le démontrent Bar-Yosef et al. ${ }^{1}$ dans ce numéro du Journal.

\section{References}

1 Bar-Yosef S, Cozart HB, Phillips-Bute B, Mathew JP, Grocott HP. Preoperative low molecular weight heparin reduces heparin responsiveness during cardiac surgery. Can J Anesth 2007; 54: 107-13.

2 Barrow RT, Parker ET, Krishnaswamy S, Lollar P. Inhibition by heparin of the human blood coagulation intrinsic pathway factor X activator. J Biol Chem 1994; 269: 26796-800.

3 Tollefsen DM, Majerus DW, Blank MK. Heparin cofactor II. Purification and properties of a heparin-dependent inhibitor of thrombin in human plasma. J Biol Chem 1982; 257: 2162-9.

4 Hirsh J, Raschke R. Heparin and low-molecular- 
weight heparin: the Seventh ACCP Conference on Antithrombotic and Thrombolytic Therapy. Chest 2004; 126(3 Suppl): 188S-203S.

5 Nurmohamed MT, Rosendaal FR, Buller HR, et al. Low-molecular-weight heparin versus standard heparin in general and orthopaedic surgery: a meta-analysis. Lancet 1992; 340: 152-6.

6 White B, Perry D. Acquired antithrombin deficiency in sepsis. Br J Haematol 2001; 112: 26-31.

7 Despotis G, Levine W, Joist JH, Joiner-Maier D, Spitznagel $\mathrm{A}$. Antithrombin III during cardiac surgery: effect on response of activated clotting time to heparin and relationship to markers of hemostatic activation. Anesth Analg 1997; 85: 498-506.

8 RxMed. Available from URL; http://www.rxmed. com/b.main/b2.pharmaceutical/b2.prescribe.top.html (accessed November 2006).

9 Despotis GJ, Joist JH, Hogue CW Jr, et al. The impact of heparin concentration and activated clotting time monitoring on blood conservation. A prospective, randomized evaluation in patients undergoing cardiac operation. J Thorac Cardiovasc Surg 1995; 110: 46-54.

10 Gibbs NM, Yim D, Weightman W, Sheminant M, Rowe $R$. The effect of excess protamine on thrombelastography in vitro. Anaesth Intensive Care 2006; 34: 579-85.

11 Cosgrove DM 3rd, Heric B, Lytle BW, et al. Aprotinin therapy for reoperative myocardial revascularization: a placebo-controlled study. Ann Thorac Surg 1992; 54: 1031-6.

12 Hunt BJ, Murkin JM. Heparin resistance after aprotinin (Letter). Lancet 1993; 341: 126.

13 Ganter MT, Monn A, Tavakoli R, et al. Monitoring activated clotting time for combined heparin and aprotinin application: in vivo evaluation of a new aprotinin-insensitive test using Sonoclot. Eur J Cardiothorac Surg 2006; 30: 278-84.

14 Smedira NG, Dyke CM, Koster A, et al. Anticoagulation with bivalirudin for off-pump coronary artery bypass grafting: the results of the EVOLUTION-OFF study. J Thorac Cardiovasc Surg 2006; 131: 686-92.

15 Koster A, Buz S, Hetzer R, Kuppe H, Breddin K, Harder $S$. Anticoagulation with argatroban in patients with heparin-induced thrombocytopenia antibodies after cardiovascular surgery with cardiopulmonary bypass: first results from the ARG-E03 trial. J Thorac Cardiovasc Surg 2006; 132: 699-700. 